\title{
The Effectiveness of Using Telemedicine to Follow-up Breast Cancer During the Covid-19 Pandemic: A Scoping Review
}

\author{
(1) Emine GERÇEK ÖTER, ${ }^{1}$ (1) Sultan ÖZKAN, ${ }^{2}$ (1) Halise ÇINAR ${ }^{3}$ \\ 'Department of Obstetrics and Gynecology Nursing, Aydın Adnan Menderes University Faculty of Nursing, Aydın-Turkey \\ 2Department of Surgical Nursing, Aydın Adnan Menderes University Faculty of Nursing, Aydın-Turkey \\ ${ }^{3}$ Department of Medical Services and Techniques, Aydın Adnan Menderes University Faculty of Söke Health Services \\ Vocational, Aydın-Turkey
}

\begin{abstract}
SUMMARY
The coronavirus disease (COVID)-19 outbreak has brought to the agenda that telemedicine (TM) services, which are rapidly spreading in the world, can be effective in reducing face-to-face medical appointments for cancer follow-up and breast cancer screening. The aim of this study is to evaluate the effectiveness of TM use in the follow-up of women diagnosed with breast cancer during the COVID-19 epidemic. The literature search was carried out in four electronic databases (PubMed, CINAHL, Science Direct, and PsycInfo) until April 14, 2020. Electronic searches started on March 22, 2020, and ended on April 14, 2020. Search terms included were tele-health or telephone and pandemic or SARS-COV-2 outbreak. Search was limited to English language and full-text articles. A total of 334 articles matching the search criteria were identified by the researchers. Five articles with no restrictions in research design met the inclusion criteria and were included in the review. In these studies, it was determined that TM services are an effective method for relieving outpatient services in breast cancer follow-up, breast imaging, screening, routine follow-up, and examinations. In addition, these studies concluded that TM services save time, increase access to care, provide improvement, and increase satisfaction levels. It was observed that the demand of women with breast cancer to benefit from TM services was affected by sociodemographic factors (advanced age and low education level, etc.). It is recommended that TM services be taken into account in the realization of individual care and follow-up services for women with breast cancer.
\end{abstract}

Keywords: Breast cancer; coronavirus disease-19; follow-up; telemedicine; treatment.

Copyright $\odot$ 2022, Turkish Society for Radiation Oncology

\section{Introduction}

Telemedicine (TM) is the use of electronic information and communication technology to provide health-care services when health-care personnel and patients are separated by physical distance.[1] TM applications can be in the form of teleconsultation, telemonitoring, and tele-expertise, and in each scenario, communication is either between health-care profes- sionals or between patients and health-care personnel.[2] It is considered as an advantage that TM applications reduce hospital visits by patients, increase access to consultation, training and consultancy services, and reduce the cost of health-care services for patients, health-care personnel, and institutions.[3] Disadvantages are noted as the elimination of the patient's physical examination, difficulty in prescribing tests and medical treatment electronically, and some-

Dr. Emine GERÇEK ÖTER

Aydın Adnan Menderes Üniversitesi Hemşirelik Fakültesi, Kadın Hastalıkları ve Doğum Hemşireliği Anabilim Dalı,

Aydın-Turkey

E-mail: eminegercekoter@adu.edu.tr 
times the unwillingness of health-care providers to pay for TM consultations. $[3,4]$

The novel coronavirus disease (COVID-19), first identified as an enveloped, positive RNA virus similar to SARS-CoV in China's Hubei Province in December 2019, later began to appear in most countries outside of China. The first case of COVID-19 was reported to the World Health Organization (WHO) on December 31, 2019. The WHO defined this disease as an epidemic on March 11.[5] The highly contagious virus quickly spread to other countries and was declared a global pandemic, affecting more than $167,492,769$ million people and killing more than 3,482,907 people. [6] Social distancing is one of the mitigating strategies to reduce the spread of COVID-19.[7] TM methods are seen as one of the most beneficial methods that can be used to provide social distance. In this process, as in many other types of cancer, there are difficulties in the management of breast cancer treatment due to the limited use of resources and the discipline of health personnel according to pandemic conditions. [3] According to the 2018 data of the WHO International Agency on Cancer for Research, the number of newly diagnosed breast cancer patients worldwide was $2,000,088$, and the number of newly diagnosed patients in Turkey in 2018 was 22,500.[8,9]

European Society of Medical Oncology (ESMO), American Society of Breast Surgeons (ASBrS), National Breast Centers Accreditation Program (NAPBC), National Comprehensive Care Network (NCCN), Cancer Commission ( $\mathrm{CoC})$, and American College of Radiology (ACR) recommended postponing non-surgical emergencies due to COVID-19 pandemic.[10,11] Guidelines developed by the ESMO, ASBrS, NAPBC, NCCN, CoC, and ACR were used as a standard guide for clinical decision-making during the 19 pandemic in hospitals around the world.[12] The main points in maintaining the treatment of patients with breast cancer have been to ensure a low risk of infection or exposure among patients and staff, to protect patients from treatment-related side effects such as immunosuppression, and from viral sources within the healthcare system.[13] In this period, in the treatment and care management of women with breast cancer, making virtual participation meetings for the treatment processes of health professionals, deciding on the process of the follow-up of the patients, identifying vulnerable patients, and communicating to discuss changes in care and treatment; the importance of deciding and implementing initiatives to identify suitable patients for remote monitoring/ follow-up and to provide data entry (where remote access is enabled) came to the fore.[14]

\section{Design}

The literature search was carried out in four electronic databases (PubMed, CINAHL, Science Direct, and PsycInfo) until April 14, 2020 (articles published in 2021 and 2020). Electronic searches started on March 22, 2020, and ended on April 14, 2020. The medical topics "Telemedicine" and "breast cancer" and "COVID-19" were used. Search terms included were tele-health or telephone and pandemic or SARSCOV-2 outbreak. Search was limited to English language and full-text articles.

\section{Methods}

Scoping review was considered the most appropriate method to approach the purpose of the designed study, as it is more flexible than the standard meta-analytic systematic review, which aims to answer specific questions regarding the identified problem. Scoping review is defined as the process of producing a comprehensive and broad overview of the area of interest. Quick reviews are accepted as an optimal approach for producing contextualized information at the point of continuing the decision-making mechanisms of health systems in acute processes.[15]

\section{Scoping Review Research Question}

How effective is the use of TM to continue breast cancer follow-up during the COVID-19 pandemic?

\section{Data Sources and Search Strategy}

A literature search was conducted in four electronic databases (PubMed, CINAHL, Science Direct, and PsycInfo) using MeSH and key terms to explore the current state of knowledge regarding the effectiveness of using TM to pursue breast cancer surveillance during the COVID-19 pandemic (articles published in 2020 and 2021). Electronic searches started on March 22, 2020, and ended on April 14, 2020. The search was done with $\mathrm{MeSH}$ keywords (tele-health * OR telemedicine ${ }^{\star}$ OR telephone ${ }^{\star}$ OR mobile phone ${ }^{\star}$ OR mphone ${ }^{*} \mathrm{OR}$ videoconferencing ${ }^{*} \mathrm{OR}$ breast cancer ${ }^{*}$ OR breast surveillance ${ }^{\star}$ OR COVID $-19 *$ SARS $-\mathrm{CoV}-2$ * OR pandemic ${ }^{*} \mathrm{OR}$ outbreak). The review follows the PRISMA-EQUATOR checklist for systematic reviews.

\section{Eligibility Criteria}

\section{Study Design}

We included studies quantitative methods (cross-sectional study and cohort study) in this review. 


\section{Population}

It was aimed to determine the status of women who were diagnosed with breast cancer before the pandemic and whose treatment process (chemotherapy, surgery, etc.) benefited from TM services to continue their treatment processes during the COVID-19 period.

\section{Concepts}

Concepts for this scoping review are studies investigating the effectiveness of TM services in maintaining follow-up of women with breast cancer in our participant population. In this study, the findings obtained from studies evaluating the effectiveness of using these services in women who want to continue their treatment using tele-medicine services because they do not want to go to hospitals due to the risk of infection during the pandemic process will be evaluated.

\section{Inclusion and Exclusion Criteria}

This review included quantitative (cross-sectional and cohort studies), English language, and full-text articles. Non-research case studies and review articles were not included. Studies examining services provided by methods other than tele-medicine were not included.

\section{Study Selection}

A two-stage screening process was used following the search in the databases. Two independent researchers scanned the titles and abstracts. In the second stage of the process, researchers who completed the review scanned the full text of the studies selected in the first stage. Full-text studies that did not meet the inclusion criteria were also excluded. Disputes were resolved through consensus discussions.

\section{Data Collection and Synthesis}

Two authors (first and second authors) independently reviewed all citations by title and abstract. When there was no consensus among researchers, citations were moved to full-text selection. In case of disagreement in the selection of the full text, it was resolved with the participation of a third author. Data were extracted independently by the first and second authors using a data extraction sheet specific to a piloted review. Data extracted included author(s), year and country of publication, design, number of participants, assessment method, purpose, and key findings. Consensus was sought and also $25 \%$ of the data were rechecked. The findings are summarized narratively, and we provide a summary of all factors reviewed in the literature in Table 1.

\section{Data Presentation}

\section{Results}

A search of databases yielded 334 articles. After removing the duplicated articles, the title and summary review were conducted for the remaining 299 articles. Articles were examined independently to identify those suitable for full-text review. Eighteen articles were included in the full-text review, and five met the inclusion criteria (Fig. 1). Among the reasons for exclusion of 13 articles are as follows: The main output of the study in four studies, the population was not suitable in five studies. There was no language compatibility in four of them.

Mella-Abarca et al. (2020)[16] conducted a TM application with video and phone calls in a hospital in Chile, where early intervention and prospective physical therapy models in breast cancer have proven to be effective in reducing postoperative breast complications. In the study, it was concluded that it is possible to apply this model. Ylldız and Öksüzoğlu's (2020)[17] study emphasized that TM can open a new era for clinics where breast cancer patients are concentrated and that TM can be a good alternative in extraordinary cases such as COVID-19. The patients stated that they were satisfied with TM, asking their questions during the pandemic process and communicating with their doctor relieved them. In the study of Zimmerman et al. (2020),[18] it was stated that the use of tele-health services in addition to the personal care of breast cancer patients will make a great contribution to optimize patient satisfaction, save time, and increase access to care during epidemic periods such as COVID-19. In the study conducted by Merz et al. (2021),[19] it was determined that the telephone tracking system is an important way to avoid hospital contact during the COVID-19 epidemic and that the majorities of women with breast cancer are satisfied with the application and recommend it. In the study of Sonagli et al. (2021)[20] in Brazil, tele-medicine was used to reduce face-to-face medical appointments in cancer follow-up and breast cancer screening during the COVID-19 epidemic. In the study, it was reported that the tele-medicine method is an easy alternative method and is used by patients on request.

\section{Discussion}

In this scoping review, we found five studies that examined the effectiveness of using TM to continue breast cancer follow-up, particularly during the COVID-19 pandemic. Our review focuses on studies that have out- 


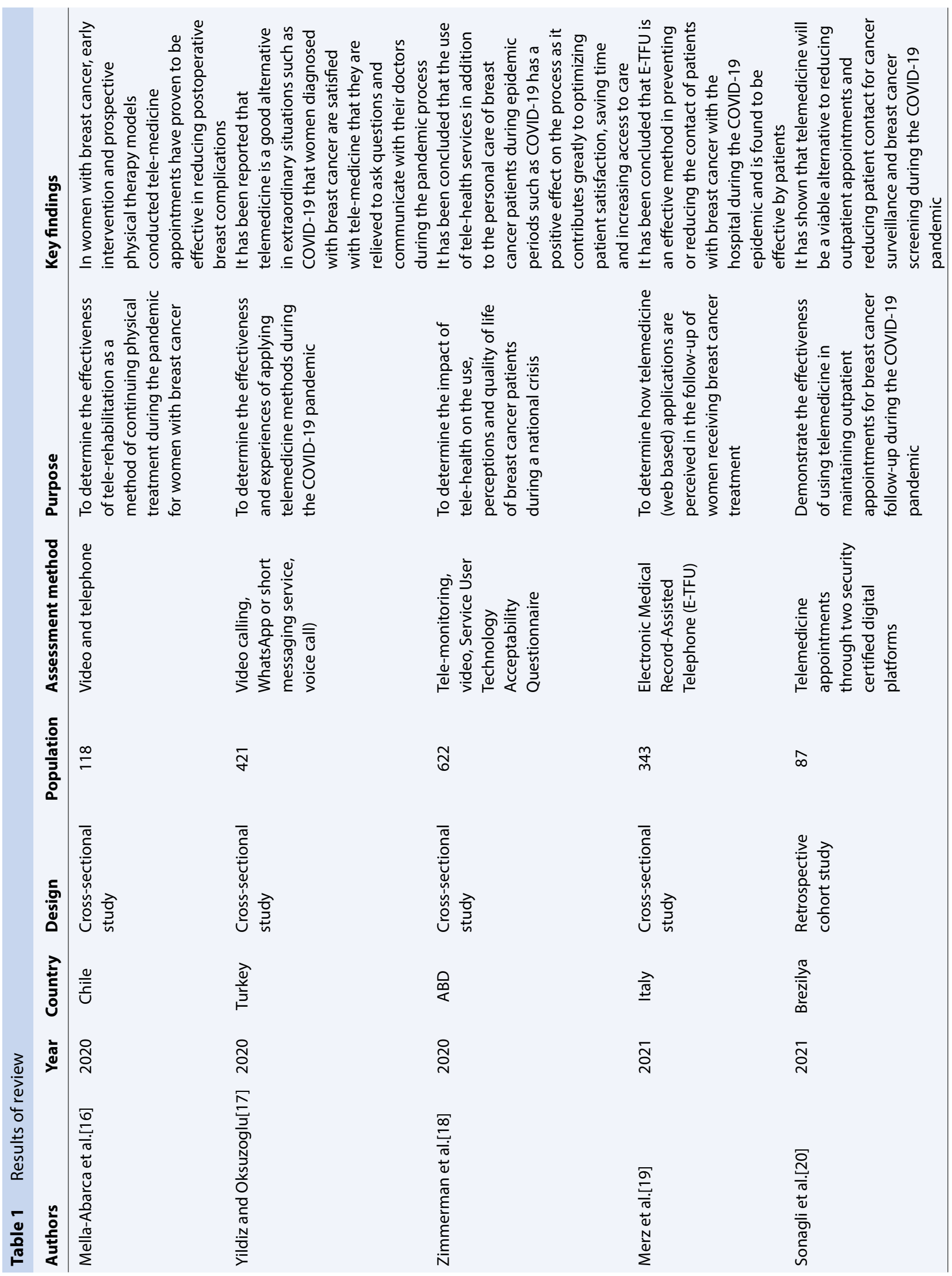




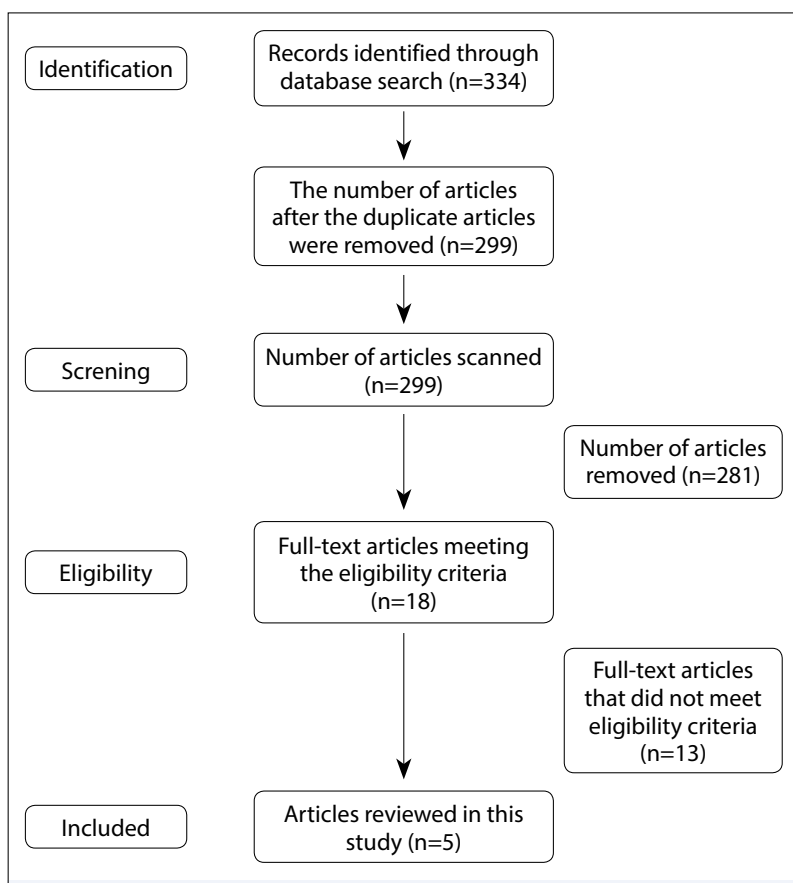

Fig. 1. PRISMA flow diagram of the study selection, including literature search.

comes such as maintaining the current level of patient satisfaction, which is one of the quality standards criteria during the pandemic, and optimizing it in changing health-related processes, creating effectiveness for both patients and health-care personnel with time management, increasing access to health-care services, and ensuring continuity of care. It is important in terms of both the perception of the necessity of using technology by health-care personnel and patients in the age of technology, and showing the benefits of using TM technologies for patients, health-care personnel, and the institution in processes such as pandemics.

The studies have demonstrated that the use of TM is increasing worldwide.[3] It has been reported that TM applications increase the access to health information and services, improve the delivery and quality of care, allow quality control of screening programs, and reduce health-care costs as well as improve health education.[21] However, there is insufficient use of TM applications, especially by individuals living in areas with low socioeconomic status, due to difficulties in accessing the internet. In addition, difficulties in communicating with individuals with low education levels are also expressed.[22] On the other hand, studies are supported by studies that TM is an important health service application that comes to the fore as it provides the delivery of care services that cannot be provided during the pandemic process. [23]
The International Society of Geriatric Oncology (SIOG) COVID-19 Working Group emphasized the importance of creating information on cancer management in older adults during the COVID-19 pandemic process, and stated that ongoing research is required to expand the knowledge on this subject. [24] However, it was also stated that the pandemic is a major hurdle and efforts are being made to increase clinical research and ensure fair access to prospective data collection on the impact and management of the process of COVID-19 in patients with cancer.[25] The University of Rochester has developed a modified telehealth geriatric assessment system as part of University of Rochester Specialized Oncology Care and Research in the Elderly.[24] It is stated that the increase in such practices globally in processes such as pandemics will contribute to the organization of follow-up, treatment, and care processes for cancer patients.[22] Our study is also of critical importance in terms of demonstrating the effectiveness and necessity of tele-medicine applications. Countries can develop specific evaluation systems for the application of tele-medicine within the scope of providing and conducting health services for cancer patients within the framework of their health policies during the pandemic process.

\section{Conclusion}

In the current review, it is seen that TM applications are effective in the follow-up of women with breast cancer in pandemics such as COVID-19, patients are largely satisfied with the application, at least with less hospital admission rates, not only for the patient but also for the health-care personnel and health-care providers during the pandemic process. Further studies will help evaluate TM efficiency by comparing TM users with face-to-face care patients. Further studies are needed to explore the application of TM outside of an emergency. Considering that the pandemic process will continue with different variances, it is of great importance to develop tele-medicine applications, especially for cancer patients, whose effectiveness and applicability have been tested in randomized controlled studies and supported by high evidence study results.

Peer-review: Externally peer-reviewed.

Conflict of Interest: The authors declare no conflicts of interest.

Financial Support: The authors received no financial support for the publication of this article. 


\section{References}

1. Sirintrapun SJ, Lopez AM. Telemedicine in cancer care. Am Soc Clin Oncol Educ Book 2018;38:540-5.

2. Aapro M, Bossi P, Dasari A, Fallowfield L, Gascón P, Geller M, et al. Digital health for optimal supportive care in oncology: Benefits, limits, and future perspectives. Support Care Cancer 2020;28(10):4589-612.

3. Gudi N, Konapur R, John O, Sarbadhikari S, Landry $\mathrm{M}$. Telemedicine supported strengthening of primary care in WHO South East Asia region: Lessons from the COVID-19 pandemic experiences. BMJ Innov 2021;7(3):580-5.

4. Hong Z, Li N, Li D, Li J, Li B, Xiong W, et al. Telemedicine during the COVID-19 pandemic: Experiences from Western China. J Med Internet Res 2020;22(5):e19577.

5. World Health Organization. WHO Director-General's Opening Remarks at the Media Briefing on COVID-19. Available at: https://www.who.int/director-general/speeches/detail/who-director-general-s-opening-remarks-at-the-mediabriefing-on-covid-19---11march-2020 Accessed May 22, 2021.

6. World Health Organization. Coronavirus disease (COVID-2019) Situation Reports. Geneva: World Health Organization; 2019. Available at: https:// www.who.int/emergencies/diseases/novel-coronavirus-2019/situation-reports Accessed April 15, 2021.

7. de Figueiredo CS, Sandre PC, Portugal LC, Mázala-deOliveira T, da Silva Chagas L, Raony Í, et al. Covid-19 pandemic impact on children and adolescents' mental health: Biological, environmental, and social factors. Prog Neuropsychopharmacol Biol Psychiatry 2021;106:110171.

8. Siegel RL, Miller KD, Jemal A. Cancer statistics, 2019. CA Cancer J Clin 2019;69(1):7-34.

9. Turkish Republic Ministry of Health. (2020) Breast Cancer Prevention, Screening, Diagnosis, Treatment and Follow-up Clinical Guide. T.R. Ministry of Health Publication Number: 1170. T.C. Ankara: General Directorate of Health Services Research, Development and Health Technology Evaluation Department, Available at: https://www.vahitozmen.com/t/wp-content/uploads/2020/07/meme-kanseri-rehberi-2020. pdf Accessed May 22, 2021.

10. Curigliano G, Cardoso MJ, Poortmans P, Gentilini O, Pravettoni G, Mazzocco K, et al. Recommendations for triage, prioritization and treatment of breast cancer patients during the COVID-19 pandemic. Breast 2020;52:8-16.

11. Vicini E, Galimberti V, Naninato P, Vento AR, Ribiero Fontana SK, et al. COVID-19: The European Institute of Oncology as a "hub" centre for breast cancer surgery during the pandemic in Milan (Lombardy Region, Northern Italy) a screenshot of the first month. Eur J Surg Oncol 2020;46:1180-1.

12. American Society of Breast Surgeons. Recommendations for Prioritization, Treatment and Triage of Breast Cancer Patients during the COVID-19 Pandemic: Executive Summary. Available at: https://www. breastsurgeons.org/docs/news/The_COVID-19_Pandemic_Breast_Cancer_Consortium_Recommendations_EXECUTIVE_SUMMARY.pdf. Accessed Jun 03, 2021.

13. Tsai HY, Chang YL, Shen CT, Chung WS, Tsai HJ, Chen FM. Effects of the COVID-19 pandemic on breast cancer screening in Taiwan. Breast 2020;54:52-5.

14. Al-Shamsi HO Alhazzani W, Alhuraiji A, Coomes EA, Chemaly RF, Almuhanna M, et al. A practical approach to the management of cancer patients during the novel coronavirus disease 2019 (COVID-19) pandemic: An international collaborative group. Oncologist 2020;25(6):e936-45.

15. Peters MD, Marnie C, Tricco AC, Pollock D, Munn Z, Alexander L, et al. Updated methodological guidance for the conduct of scoping reviews. JBI Evid Synth 2020;18(10):2119-26.

16. Mella-Abarca W, Barraza-Sánchez V, Ramírez-Parada K. Telerehabilitation for people with breast cancer through the COVID-19 pandemic in Chile. Ecancermedicalscience 2020;14:1085.

17. Yildiz F, Oksuzoglu B. Teleoncology or telemedicine for oncology patients during the COVID-19 pandemic: The new normal for breast cancer survivors? Future Oncol 2020;16(28):2191-5.

18. Zimmerman BS, Seidman D, Berger N, Cascetta KP, Nezolosky M, Trlica K, et al. Patient perception of telehealth services for breast and gynecologic oncology care during the COVID-19 pandemic: A single center survey-based study. J Breast Cancer 2020;23(5):542-52.

19. Merz V, Ferro A, Piras EM, Alberto Z, Caffo O, Messina C. Electronic medical record-assisted telephone follow-up of breast cancer survivors during the COVID-19 pandemic: A single institution experience. JCO Oncol Pract 2021;17(1):e44-52.

20. Sonagli M, Neto RC, Leite FP, Makdissi FB. The use of telemedicine to maintain breast cancer follow-up and surveillance during the COVID-19 pandemic. J Surg Oncol 2021;123(2):371-4.

21. Ortega G, Rodriguez JA, Maurer LR, Witt EE, Perez N, Reich A, et al. Telemedicine, COVID-19, and disparities: Policy implications. Heal Policy Technol 2020;9(3):368-71.

22. Araujo DV, Watson GA, Siu LL. The day after 
COVID-19 time to rethink oncology clinical research. JAMA Oncol 2021;7(1):23-4.

23. Bashshur R, Doarn CR, Frenk JM, Kvedar JC, Woolliscroft JO. Telemedicine and the COVID-19 pandemic, lessons for the future. Telemed J E Health 2020;26(5):571-3.

24. Battisti NM, Mislang AR, Cooper L, O’Donovan A, Audisio RA, Cheung KL, et al. Adapting care for older cancer patients during the COVID-19 pandemic: Recommendations from the international society of geriatric oncology (SIOG) COVID-19 working group. J Geriatr Oncol 2020;11(8):1190-8.

25. Gill S, Hao D, Hirte H, Campbell A, Colwell B. Impact of COVID-19 on Canadian medical oncologists and cancer care: Canadian association of medical oncologists survey report. Curr Oncol 2020;27(2):71-74. 